\title{
Building a Distributed Digital Library System Enhancing the Role of Metadata
}

\author{
Gianmaria Silvello \\ Department of Information Engineering \\ University of Padua \\ Via Gradenigo, 6/B \\ 35131 Padova (Italy) \\ silvello@dei.unipd.it
}

\begin{abstract}
In this work we present a methodology that maps a tree data structure in a nested sets organization; the aim of this methodology is to provide an effective and efficient way to manage and exchange descriptive archival metadata in a distributed environment. We consider archival metadata as a basis for our study because of their challenging nature and the management and exchange difficulties they present. Furthermore, we describe a distributed Digital Library System (DLS) architecture that would successfully apply the designed methodology. We also introduce future investigations concerning the definition of nested sets organization as an alternative way to manage hierarchical data.
\end{abstract}

Keywords: Distributed Digital Library Systems, Digital Archive Systems, OAI-PMH, Nested Sets Model

\section{INTRODUCTION}

The role of Digital Library Systems (DLSs) in collecting, managing, sharing and preserving our cultural heritage is increasingly prominent in several contexts. DLSs are becoming the fundamental tool for pursuing interoperability between different cultural organizations such as libraries, archives and museums. Collecting and managing the resources of these organizations is fundamental for providing wide, distributed and open access to our cultural heritage.

In this wide and heterogeneous scenario, interoperability is the most relevant issue that a DLS has to face. In a distributed environment the first problem is interoperability between different information systems; a DLS must be able to collect resources shared by a wide number of different systems without compromising their autonomy and independence. We consider a wider context that includes resources of different organization types; in this context the interoperability issue is emphasized by two main necessities. These are the designing of a unique access point to several resources widely different in nature and the cooperation between different information systems.

In this work we consider a challenging kind of information resource: archival documents. When archives are considered, interoperability between the archives themselves, between archival resources and between archival and other types of resources must be taken into account. In the work we have been carrying out we have underlined that DLS technologies need to be revisited to be well-suited and successfully applied to the management of archival metadata and digital objects [1, 2]. In this paper we briefly describe the nature of archival resources, we introduce a methodology based on a nested sets organization able to manage and exchange the archival metadata preserving their whole informative power and we present a DLS architecture that enables them to be included in a DLS. Moreover, we introduce possible future investigations concerning the generalization and formalization of the nested sets organization methodology; in particular, we present the issues that must be considered in order to define the nested sets organization as a way of managing hierarchical data. 
The paper is organized as follows: in Section 2 we present the background, projects and initiatives that constitute the context in which this work has been carried out. In Section 3 a brief analysis of archive peculiarities is reported. Section 4 presents the nested sets methodology applied to archival descriptive metadata. Section 5 presents an applicative scenario in which the nested sets methodology can be applied; this applicative scenario is a distributed DLS architecture which we defined in order to share and develop advanced services on archival metadata in a distributed environment. In Section 6 we present future investigations concerning the nested sets methodology.

\section{BACKGROUND}

In order to provide wide access to large and broad collections of digital resources and to address interoperability issues, several initiatives have been instituted. The DELOS Network of Excellence on Digital Libraries ${ }^{1}$ has proposed and developed a reference model for laying the foundations of digital libraries [3] which takes into account the perspectives and needs of different cultural heritage institutions and provides a coherent view on the main concepts which constitute the universe of digital libraries in order to facilitate co-operation among different systems.

The "European Commission Working Group on Digital Library Interoperability", active from January to June 2007, had the objective of providing recommendations for both a short-term and a long-term strategy towards "the setting up of the European Digital Library as a common multilingual access point to Europe's distributed digital cultural heritage including all types of cultural heritage institutions" [11]. In particular, the recipient of these recommendations is the Europeana thematic network ${ }^{2}$, which is a project launched in July 2007 with the aim of addressing the interoperability issues among European museums, archives, audio-visual archives and libraries towards the creation of the "European Digital Library".

Interoperability between different systems has been promoted by the Open Archives Initiative $(O A I)^{3}$ through Open Archives Initiative Protocol for Metadata Harvesting (OAI-PMH) [20], a flexible and lightweight protocol for metadata harvesting, which is becoming the de-facto standard in metadata exchange in distributed environments. This protocol permits metadata harvesting between different repositories in a straightforward fashion, in order to create aggregated metadata collections and to enable the creation of advanced services on them. At the same time Dublin Core $(D C)$, a tiny and lightweight metadata format, is becoming the preponderant means for the exchange of information in a wide distributed environment. Indeed, the characteristics of DC have enabled it to address several interoperability problems and it has been chosen as the minimum common denominator in the OAI-PMH environment. Libraries have been using the couple OAI$\mathrm{PMH}$ and $\mathrm{DC}$ for a relatively long time with good results [19].

In fact, in order to develop a large Digital Library ( $D L)$ among all initiatives, two relevant European initiatives are The European Library portal ${ }^{4}$ and Digital Repository Infrastructure Vision for European Research (DRIVER) $)^{5}$ wich both enjoy the benefits of OAI-PMH. The European Library is a free service that offers access to the resources of the 48 national libraries of Europe in 20 languages. The goal of The European Library is to create a single access point to all the European national libraries. The European Library project offers a concrete integration possibility based on OAI-PMH, used to collect the catalogue records of national libraries. Furthermore, the TELplus project ${ }^{6}$ will form another building block of the European Digital Library and is aimed at strengthening, extending and improving The European Library service. In particular, to contribute to interoperability among different organizations cooperating in The European Library, it aims to improve and enhance the adoption of OAI-PMH as a means of integration.

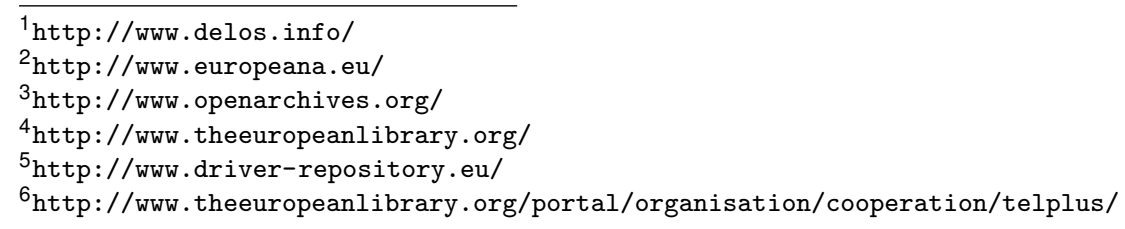


DRIVER is a European project whose goal is to develop a pan-European Digital Repository Infrastructure by integrating existing individual repositories from European countries and developing a core number of services, including search, data collection, profiling and recommendation [4]. DRIVER emphasizes the implementation of nominal, globally accepted standards in a real-life system, with a focus on metadata exchange, in particular using OAI$\mathrm{PMH}$. One of the Digital Library application components provided by DRIVER is an OAI-Publisher Service; in this way DRIVER services operate upon the aggregated content of existing institutional OAl repositories.

Nevertheless, in the archive context neither general interoperability efforts nor the adoption of specific solutions such as OAI-PMH are common and widespread; this tends to exclude archival documents from forming a valuable part of the cultural heritage managed by a DLS.

\section{PECULIARITIES OF ARCHIVES}

An archive is the trace of the activities of physical people or juridical organizations in the course of their business. Archives have been preserved because of their continued value over time. Archives have to keep the context in which their documents have been created and the network of relationships among them in order to preserve their informative content and provide understandable and useful information over time. In this way archives are able to preserve the provenance of their documents; the preservation of provenance of digital resources is an important issue currently being investigated by the scientific community [15] and must be considered as a key feature of a DLS; it is through provenance information that authenticity can be demonstrated, and the history of archival documents can be preserved.

Archival descriptions have to reflect the peculiarities of the archive, retain all the informative power of a record and keep trace of the provenance and original order in which resources have been collected and filed by archival institutions [10]. Indeed, archivists seek to group and describe all the archives created by the same organization together, and call this by its French name "fonds". As indicated in [6], the fonds should be viewed primarily as an "intellectual construct", the conceptual "whole" that reflects an organic process in which a records creator produces or accumulates series of records. In this context, provenance becomes a fundamental principle of archives; the principle of the "respect des fonds" which dictates that resources of different origins be kept separate to preserve their context [7] suggests that maintaining provenance leads archivists to evaluate records on the basis of the importance of the creator's mandate and functions, and fosters the use of a hierarchical method where the hierarchical structure of the archive expresses the relationships and dependency links among the records of the archive by using what is called the archival bond. Archival bonds, and thus relations, are constitutive parts of archival resources: if an archival resource were taken out from its context and lost its relations, its informative power would also be considerably affected.

Archival descriptive metadata are the foremost digital resources shared by the archives. Indeed, most archival documents are not available in digital form, but they are described and represented by metadata; sometimes archival resources are metadata themselves. In the archival context metadata are called archival descriptive metadata and express the archival descriptions.

The use of metadata allows us to exploit DLS technologies and data exchange protocols and apply them to the archival descriptions. Archival description metadata should meet the following three main requisites:

1. Context: archival description metadata have to retain information about context of a given record, such as the relations between records and the production environment, as stated by the respect des fonds principle discussed above.

2. Hierarchy: archival description metadata have to reflect the archive organization which is described in a multi-leveled fashion.

3. Variable granularity: archival description metadata have to facilitate access to the requested items, which may belong to different hierarchical levels, with the desired degree of detail and without requiring the whole hierarchy to be accessed. 
The only standard defined for archival descriptive metadata is the Encoded Archival Description $(E A D)$ metadata format. EAD reflects the archival structure and holds relations between entities in an archive. In addition, EAD encourages archivists to use collective and multilevel description. On the other hand, EAD allows for several degrees of freedom in tagging practice, which may turn out to be problematic in the automatic processing of EAD files. The EAD permissive data model may undermine the very interoperability it is intended to foster. Moreover, EAD files are heavy and difficult-to-move; it has been underlined $[13,16]$ that the EAD metadata standard is not well-suited for use in a distributed environment.

\section{A METHODOLOGY FOR MANAGING AND SHARE EAD FILES}

Different solutions have been studied to permit archival descriptive metadata exchange in a distributed environment. The proposed solutions suggest the couple DC and OAI-PMH as the means to enable the sharing of archival descriptive metadata and to map EAD files in shareable metadata format. The solution proposed in [18] suggests mapping an EAD file into many tiny and easy-to-move DC metadata. In this approach every DC metadata record generated contains a link to the original EAD file. With this approach there is a strong dependency with the original EAD file that undermines the exchange possibilities of the DC metadata [17]. The solution we proposed in [9] defines a methodology that joins and exploits the characteristics of OAI-PMH and DC. This methodology enables archive hierarchy to be expressed and meaningful relations between archival entities to be preserved by levering the role of the OAl sets. The main idea is to map the archive hierarchy into a combination of OAI sets and DC metadata records.

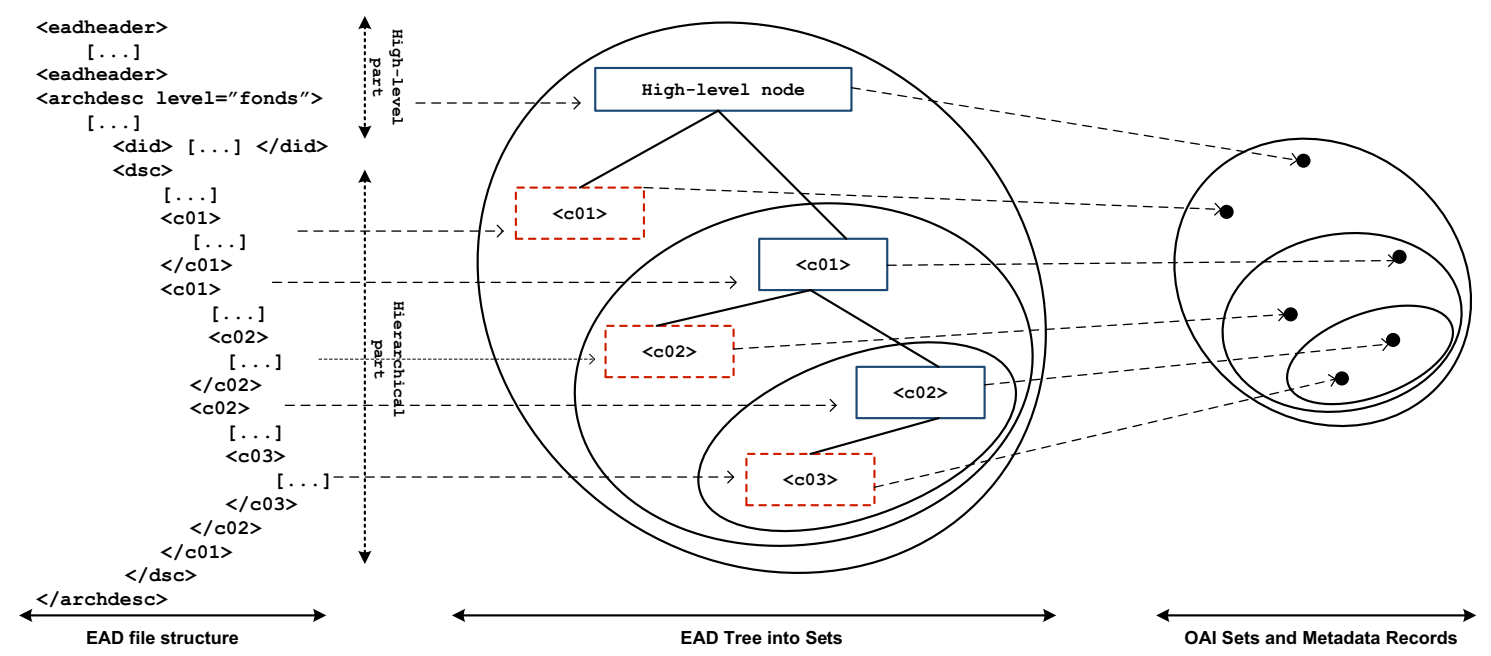

Figure 1: Mapping EAD metadata into OAI Sets and DC metadata records.

In Figure 1 we take up the EAD file structure showing how its tree representation can be mapped in a collection of sets ${ }^{7}$. This methodology permits the exchange of archival descriptive metadata in a distributed environment facing interoperability problems and the maintenance of the whole archival informative power of the metadata. We propose a methodology to map the structure of EAD files into several DC metadata records and OAI sets. As far as the mapping of the actual content of EAD items into DC records is concerned, we adopt the mapping proposed by Prom and Habing [18]. We differ from [18] in the way in which the structure of EAD files is translated into OAI sets and DC records. Our methodology ${ }^{8}$, shown in Figure 1, is constituted by three main steps:

1. OAl sets: creation of an OAI set for each internal node of the tree.

\footnotetext{
${ }^{7}$ Figure 1 has been reproduced from [9].

${ }^{8}$ To understand this methodology it is worthwhile defining two fundamental characteristics of tree data structure: internal and external nodes. An internal node is defined as a node having at least one child, instead an external node is defined as a node without children [14].
} 
2. Metadata set record: a metadata record for each set constituted in step one must be created; we call these records metadata set records because they are built contextually with the OAl set.

3. Metadata record: a metadata record for each external node of the tree must be created.

The proposed solution addresses the shortcomings of EAD when it has to be used in a distributed environment and with variable granularity access to the resources. Indeed, EAD items are mapped into different DC metadata records which are shareable metadata, and natively supported by $\mathrm{OAI}-\mathrm{PMH}$. Furthermore, context and hierarchy are preserved and expressed in a straightforward manner exploiting the native functionalities of OAI-PMH and DC metadata format. Indeed, the organization into OAI sets reflects archive hierarchy and each metadata record also maintains in its header the membership information which is essential for going up again to related entities and to express contextual information. The proposed solution also addresses variable granularity, indeed a particular archival metadata can be reached without visiting the whole hierarchy.

The proposed nested sets methodology is well-suited to managing and exchanging archival metadata in a distributed environment and it finds an application in the distributed DLS architecture presented in the next section.

\section{A DISTRIBUTED DIGITAL LIBRARY SYSTEM ARCHITECTURE}

A DL aimed at collecting and managing archival resources has to face the complex nature of archives; archive issues can be classified under the interoperability issue and the heterogeneity issue. Interoperability as we have seen is related to the sharing problems of archival resources in a distributed environment; heterogeneity is related to the broad differences between archives and archival resources. Indeed, a DLS has to consider a large number of different archives distributed in a geographical area; each archive exposes a large number of metadata that has to be collected and managed preserving their whole informative power and thus a lot of additional information as well as the metadata themselves.

The constitution of a DLS whose goal is to put archival resources together must take into account the structure and the size of the participating archives. Archives preserve resources that are unique and valuable, thus also small and medium archives need to participate in the system, thus providing important contributions. Usually, independent, private or public archives keep archival metadata without sharing them and this prevents the offering of common advanced services on metadata; one of the goals of a DLS is to provide advanced services on archival metadata. DLSs are service-oriented and can be composed of independent sub-systems that cooperate together to supply the required Digital Library functionalities. Moreover DLSs aim to strengthen integration and interoperability between different systems. The design of a DLS architecture must take into consideration several issues: it has to preserve archive autonomy and gather their metadata to perform advanced services. On the one hand we have to guarantee the bodies maintain archive management autonomy [1]. On the other, we have to build central coordination that has an integrated vision of the archives participating in the system. The added value of this DLS architecture is that it shares metadata exploiting Digital Library advances that can be integrated with and adapted to preexisting systems using different technologies. The result is a scalable, flexible and widely-adoptable architecture for sharing information in a distributed environment [8].

The developed DLS architecture exploits the characteristics of the protocol OAI-PMH based on the distinction between Data and Service Provider and the DC metadata format. The DLS architecture we designed is symmetric in sharing and managing both archival descriptive metadata and authority files treated as metadata too. Indeed, archives act as Data Provider by exposing their descriptive metadata and also as a Service Provider by harvesting the authority files exposed by the central Digital Library. The central Digital Library acts in the same way as a Data Provider furnishing authority files and as Service Provider harvesting archival descriptive metadata. Moreover, the central Digital Library acts as the central authority that constitutes authority files. 
The nested sets methodology is a constitutive part of the DLS we propose to share, collect and manage archival resources; this methodology enables several aspects of the interoperability issue to be addressed.

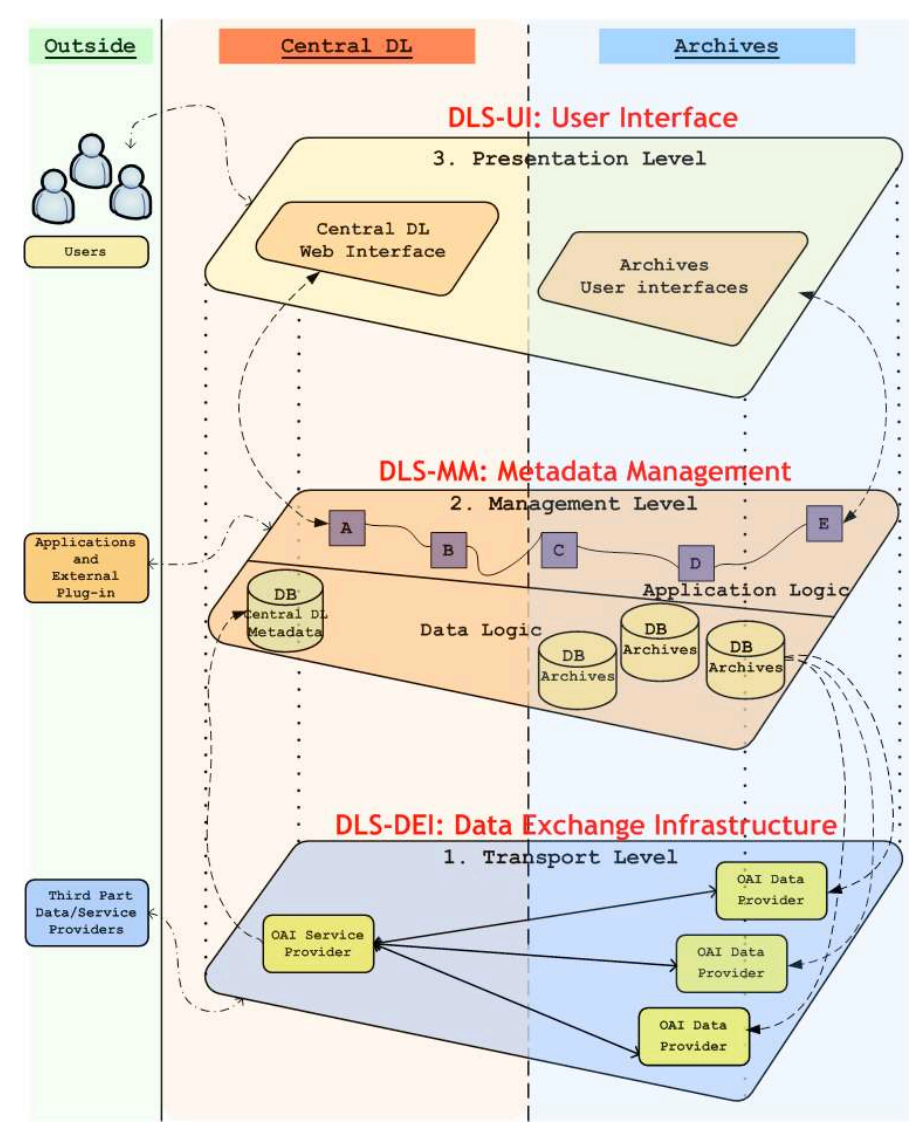

Figure 2: DLS Distributed Architecture

The DLS is developed as a three-layer architecture, composed of the metadata transport layer, the metadata management layer and the presentation layer. The transport layer represents the DLS transport infrastructure based on OAI-PMH. The archives participating in the system act as Data Providers providing archival metadata, whereas the central Digital Library acts as a Service Provider that harvests metadata. As stated above, archival metadata have to retain context and hierarchy information; we addressed this issue thanks to a methodology that combines OAI-PMH sets and DC as stated in the previous section. In order to retain this useful and fundamental information the Service Provider has to harvest not only the metadata but also the whole set organization of the Data Provider. Selective harvesting is an OAI-PMH native procedure and it permits effective metadata harvesting that preserves archival information. In this way the archive organization expressed through sets and metadata is recreated in the Service Provider, thus enabling the realization of advanced services on fully expressive archival metadata. The architecture is open to third party OAI-PMH components that for example can harvest the central Digital Library Service Provider.

At the second layer we find the management level called DLS-MM, which is composed of an Application Logic part and a Data Logic part. By the use of Application Logic we can develop advanced services both on harvested metadata owned by the central Digital Library and on the metadata of the archives. The applications developed for the DLS can be used on central Digital Library metadata index and on archive metadata too; indeed they are independent of the transport infrastructure. Thanks to this organization, adding a third-party service to the system will be almost effortless. The Application Logic works on the metadata managed by the Data Logic composed of a central database and a set of distributed databases owned by the archives. DLS-MM Data 
Logic preserves and manages the physical data of the system; so this sub-layer manages archive data and central Digital Library archive data as well.

At the third level we have the presentation layer called DLS-UI constituted by the user interfaces. The system presents two main interfaces: the first is a general-purpose interface dedicated to a generic user-type such as archivists, historical researchers, public administrations or private organizations that will use the advanced services available in the DLS; the second is dedicated to specialized users who through this interface can add, remove or update archival metadata.

\section{FINAL REMARKS AND FUTURE WORKS}

In this work we presented a DLS architecture which is able to share, collect and manage metadata in a distributed environment; lightness and scalability of this architectural solution have been shown. Our studies and the designed solutions have produced original contribution in the DL field by proposing an effective solution to handle heterogeneous digital resources.

The research results presented in this paper have been reached during the first year of the $\mathrm{PhD}$ of the author; for the future of this work, a new investigation theme concerns the evaluation of the designed methodology that permits the mapping of a tree into a nested sets organization. In the presented case the methodology based on nested sets has been used to solve a specific applicative problem (manage and exchange EAD files); it would be interesting to generalize the methodology in order to evaluate its possible applications in a wider spectrum of problems. The intuitive graphic representation of a tree as an organization of nested sets was used in [14] to show different ways to represent tree data structure and in [5] to explain an alternative way to solve in SQL language recursive queries over trees. However, nested sets representation of tree data structure has not been formalized and generalized yet; a development of this work envisions the formalization of this methodology that we call: "nested sets model". The definition of the nested sets model requires the study of the set theory in relation to the considered context. We are expected to define the nested sets model starting from the axiomatic set theory; we will formulate, in the context of nested sets model, some of the properties and operations of sets, such as the Zermelo-Fraenkel axioms and the axiom of choice. [12] states that experience has shown practically all notions used in mathematics can be defined, and their mathematical properties derived, in the set axiomatic system. In this sense, the axiomatic set theory serves as a satisfactory foundation for our model based on nested sets.

The formalization of the model will start from an evaluation based on set theory of the mapping methodology. The next steps will involve a redefinition of the methodology and a formal definition of the nested sets model. Afterwards, we will be able to define the meaning of the basic set operations as union, intersection, difference and symmetric difference in the nested sets model, in order to manipulate tree data structure and enable a new way to perform operations on hierarchical data. Throughout the nested sets model it will be possible to design new possibilities in managing, indexing and accessing hierarchical data such as the XML files or define a more effective method for solving recursive queries in relational databases.

\section{ACKNOWLEDGEMENTS}

The author would like to thank his supervisors Maristella Agosti and Nicola Ferro at the University of Padua, Department of Information Engineering. The study is partially supported by the TELplus Targeted Project for digital libraries, as part of the eContentplus Program of the European Commission (Contract ECP-2006-DILI-510003). The work of Gianmaria Silvello was partially supported by a grant from the Italian Veneto region.

\section{Bibliography}

[1] M. Agosti, N. Ferro, and G. Silvello. An Architecture for Sharing Metadata among Geographically Distributed Archives. In C. Thanos, F. Borri, and L. Candela, editors, DELOS Conference, volume 4877 of Lecture Notes in Computer Science, pages 56-65. Springer, 
Heidelberg, Germany, 2007.

[2] M. Agosti, N. Ferro, and G. Silvello. Proposta metodologica e architetturale per la gestione distribuita e condivisa di collezioni di documenti digitali. Archivi, 2(2):49-73, December 2007.

[3] L. Candela, D. Castelli, N. Ferro, G. Koutrika, C. Meghini, P. Pagano, S. Ross, D. Soergel, M. Agosti, M. Dobreva, V. Katifori, and H. Schuldt. The DELOS Digital Library Reference Model. Foundations for Digital Libraries. ISTI-CNR at Gruppo ALI, Pisa, Italy, November 2007.

[4] L. Candela, D. Castelli, P. Manghi, and P. Pagano. Enabling Services in Knowledge Infrastructures: The DRIVER Experience. In M. Agosti, F. Esposito, and C. Thanos, editors, Post-proceedings of the Third Italian Research Conference on Digital Library Systems (IRCDL 2007), pages 71-77. ISTI-CNR at Gruppo ALI, Pisa, Italy, November 2007.

[5] J. Celko. Joe Celko's SQL for Smarties: Advanced SQL Programming. Morgan Kaufmann, 2000.

[6] T. Cook. The Concept of Archival Fonds and the Post-Custodial Era: Theory, Problems and Solutions. Archiviaria, 35:24-37, 1993.

[7] L. Duranti. Diplomatics: New Uses for an Old Science. Society of American Archivists and Association of Canadian Archivists in association with Scarecrow Press, 1998.

[8] N. Ferro and G. Silvello. A Distributed Digital Library System Architecture for Archive Metadata. In 4th Italian Research Conference on Digital Libraries (IRCDL 2008). In print, 2008.

[9] N. Ferro and G. Silvello. A Methodology for Sharing Archival Descriptive Metadata in a Distributed Environment. In B. Christensen-Dalsgaard et al., editor, Proc. 12th European Conference on Research and Advanced Technology for Digital Libraries (ECDL 2008), pages 268-279. Lecture Notes in Computer Science (LNCS) 5173, Springer, Heidelberg, Germany, 2008.

[10] A. J. Gilliland-Swetland. Enduring Paradigm, New Opportunities: The Value of the Archival Perspective in the Digital Environment. Council on Library and Information Resources, 2000.

[11] S. Gradmann. Interoperability of Digital Libraries: Report on the work of the EC working group on DL interoperability. In Seminar on Disclosure and Preservation: Fostering European Culture in The Digital Landscape. National Library of Portugal, Directorate-General of the Portuguese Archives, Lisbon, Portugal, September 2007.

[12] K. Hrbacek and T. Jech. Introduction to Set Theory. Marcel Dekker, Inc., New York. New York, 1978.

[13] K. Kiesling. Metadata, Metadata, Everywhere - But Where Is the Hook? OCLC Systems \& Services, 17(2):84-88, 2001.

[14] D. E. Knuth. The Art of Computer Programming, third edition, volume 1. Addison Wesley, 1997.

[15] L. Moreau, P. Groth, S. Miles, J. Vazquez-Salceda, J. Ibbotson, S. Jiang, S. Munroe, O. Rana, A. Schreiber, V. Tan, and L. Varga. The Provenance of Electronic Data. Commununications of the ACM, 51(4):52-58, 2008.

[16] C. J. Prom. Does EAD Play Well with Other Metadata Standards? Searching and Retrieving EAD Using the OAI Protocols. Journal of Archival Organization, 1(3):51-72, 2002.

[17] C. J. Prom. Reengineering Archival Access Through the OAl Protocols. Library Hi Tech, 21(2):199-209, 2003.

[18] C. J. Prom and T. G. Habing. Using the Open Archives Initiative Protocols with EAD. In G. Marchionini and W. Hersch, editors, Proc. 2nd ACM/IEEE Joint Conference on Digital Libraries, (JCDL 2002), pages 171-180. ACM Press, New York, USA, 2002.

[19] C. J. Prom, C. A. Rishel, S. W. Schwartz, and K. J. Fox. A Unified Platform for Archival Description and Access. In E. M. Rasmussen, R. R. Larson, E. Toms, and S. Sugimoto, editors, Proc. 7th ACM/IEEE Joint Conference on Digital Libraries, (JCDL 2007), pages 157166. ACM Press, New York, USA, 2007.

[20] H. Van de Sompel, C. Lagoze, M. Nelson, and S. Warner. The Open Archives Initiative Protocol for Metadata Harvesting (2nd ed.). Technical report, Open Archive Initiative, p. 24, 2003. 